\title{
PROSPECTUS FOR THE SOLAR MAXIMUM YEAR
}

\section{(Invited Report)}

\author{
H. ZIRIN and K. TANAKA \\ California Institute of Technology, Pasadena, California, U.S.A.
}

The study of solar activity requires evolutionary and synoptic observations which would be benefitted by world-wide coordination. An international cooperative effort for coordinated study of solar activity during the period April 1, 1979November 1, 1980 has been proposed by people in the solar physics community, and a working group of Commission 10 of the IAU has been established to develop the program, which has been named the Solar Maximum Year or SMY. To provide quick response and close coordination, the SMY program will be organized on the working-scientist and observatory level, but with the cooperation of national organizations.

The problems to be attacked in the SMY have been focussed on the following four:

1. What is the pattern of development of a solar active region and its interaction with other magnetic centers?

What are the developmental characteristics that lead to solar flares?

2. What is the relation of active regions to the general large scale solar fields and the reversal of the general solar field?

3. What are all the solar inputs to the solar wind and interplanetary magnetic field?

4. What are the detailed mechanisms of solar flares?

Examples of required observations for these programs are shown in the Table "Proposed Problems in SMY". For the problem (1), particularly for the study of flare build-up processes, the importance of 24-hour monitoring of the selected active region by high resolution white light photographs may be pointed out in view of the rapid evolution of sunspots in association with major flare activity which is concentrated in a short period. Such observations have never been attempted in the past. Also detailed evolutionary study of the velocity field in the active region would be important as close association of a particular kind of velocity such as shear flow with flares has been reported. It is necessary to distinguish horizontal flow from vertical flow as well as to investigate the height variation. The problem (2) may not be completed during the short time period of the SMY; however, since such work would be particularly important to understanding the physics of the solar cycle, the SMY would provide a good opportunity to start the prolonged observation.

Although SMY is organized by solar people with a focus on the Sun, it is 
obvious that there is great potential in cooperation with the International Magnetospheric Study, the last portion of which will overlap with the SMY. It would seem sensible to use our close watch on the sun to inventory the solar inputs to the solar wind and determine their effect on the magnetosphere. For this purpose, a continuous watch of the Sun over a few solar rotations would be necessary, as well as the continuous measurement of the solar wind at the same period. There may be at least three possible solar inputs to the solar wind: (1) coronal transients, (2) coronal holes, (3) flares. Although these are best observed in the EUV, and soft X-ray regions, it would be also possible to detect these inputs from the ground-based observation only.

Any ground-based program will be greatly enhanced in value by spacecraft measurements and vice versa. From this point of view it should be emphasized to make detailed programs on cooperative bases with any planned satellites and rockets. Particular attention should be paid to the SMM. There is special interest in high resolution soft X-ray and EUV images, and hard X-ray monitoring.

For successful coordinated observations there are several things to be prepared. The working group is now engaged in the following problems:

1. An inventory of solar observatories which are willing to participate in the SMY and their potential contribution.

2. A detailed scientific program identifying specific problems which benefit from specific cooperative and long-term observations. An attempt will be made to fit the capabilities of the various observatories to such programs and divide the responsibilities among them. Observatories can contribute by observing at times when others cannot observe, or by carrying out simultaneous observations of additional parameters.

3. A plan for alerts and identification of interesting active centers or magnetic configurations.

4. How can the data best be exchanged and made available for analysis?

It is hoped to publish a solar newspaper every 27 days in which informal communication of the progress of our works will be made available to the various workers. Preliminary publishing of photographs and data may help further data exchanges although we need unique and new ideas on the data exchange problem.

Four years are available before the beginning of this program. We hope to identify needed instrumentation and stimulate its development by cooperating groups; hopefully pilot programs will be carried out. From a practical point of view it would be desirable that the SMY merges with the FBS for a given period of time at the initial stage. 


\section{Proposed Problems in SMY}

1. Pattern of development of $A R$;

its interaction with other $A R$;

developmental characteristics leading to flares

needed: all high resolution

white light ( 24 hours)

$\mathrm{H} \alpha$ (24 hours)

magnetogram and/or high field Zeeman measures (every 2 hours)

velocity field (horizontal and vertical) and its height variation (every 2 hours)

radio (including polarization)

soft X-ray and EUV images.

2. Relation of AR development to the general large-scale fields

needed:

Full disk $\mathrm{H} \alpha$

full disk magnetogram (at least every 12 hours)

soft X-ray and EUV image.

3. All inputs to the solar wind over a given period

2 solar rotations (or more) coverage. Candidates for solar inputs:

a. coronal transients - coronagraph in space; how

many prominences erupt per day?

needed: $\mathrm{H} \alpha$ disk patrol

limb patrol (coronagraph)

radio coronal patrol

b. coronal holes--soft X-ray, EUV

ground-based observations (He I $10830 \AA$ filtergrams)

c. flares.

4. Detailed mechanism of flare
a. same as in 1 .
b. high resolution radio interferometry
c. high resolution hard X-ray observation
d. gamma ray spectroscopy
e. real-time magnetogram. 